\title{
PARAMETER UNCERTAINTY ANALYSIS IN ENVIRONMENTAL RISK ASSESSMENT CAUSED BY HAZARDOUS CHEMICAL ACCIDENT
}

\author{
LIU, H. L. ${ }^{1,2}-$ LIU, N. ${ }^{3}-$ SHEN, F. ${ }^{1}-$ MA, J. J. ${ }^{1 *}$ \\ ${ }^{1}$ College of Territorial Resources and Tourism, AnHui Normal University, Wuhu 241000, China \\ ${ }^{2}$ College of Computer Science and Engineering, AnHui University of Science and Technology, \\ HuaiNan 232001, China
}

${ }^{3}$ School of Foreign Languages, AnHui University of Science and Technology, HuaiNan 232001, China

*Corresponding author

e-mail: jinjima@mail.ahnu.edu.cn, liuhl@aust.edu.cn; phone: +86-554-666-8604

(Received 23 ${ }^{\text {rd }}$ Apr 2019; accepted $4^{\text {th }}$ Jul 2019)

\begin{abstract}
Environmental risk assessment is an important part of environmental management research. However, due to uncertainties in the environmental risk system, assessment results may be partially incomplete. This study takes the leakage accident of liquid ammonia as a typical case. By combining the method of Morris parameter sensitivity analysis with the Latin Hypercube Sampling, the probability sampling analysis is conducted on a group of parameters that have the greatest impact on the output of the accident consequence calculation model, as well as a set of radius values for lethal range caused by toxic gas and its probability distribution function are obtained. based on the probability distribution, the author took advantage of the GIS tool to conduct a comprehensive analysis, thereby calculating the indexes that characterize the status of regional environmental risk. The research ideas proposed in this paper have effectively improved the rationality of the selection of the parameters in the uncertainty analysis. Furthermore, the assessment results based on the uncertainty analysis can cover all possible accident scenarios, which not only comprehensively reflects the risk status of emergency environmental accidents, but also provides more scientific and reliable information for environmental management.
\end{abstract}

Keywords: ammonia leakage accident, Latin Hypercube Sampling, Morris sensitivity analysis, individual risk, social risk

\section{Introduction}

Potential hazardous chemical accidents pose serious threat to surrounding area. With the enhancement of public awareness of environmental protection, people realize that it is necessary to establish a scientific methodology system of "environmental risk assessment" for potential accident areas, so that environmental disasters can be effectively prevented (Shao et al., 2013; Hahn et al., 2010). In a broad sense, environmental risk assessment refers to the process of management and decisionmaking based on the assessment of possible losses to the hazards (including natural disasters) caused or triggered by various social economic activity to human health, social economy, and ecosystem and so on. In a narrow sense, environmental risk assessment usually refers to the probability estimation to the impact degree of toxic and harmful substances (including chemicals and radioactive substances) on human health and ecosystem, and puts forward plans and countermeasures for reducing environmental risk (Lu et al., 1999; Hansen et al., 2016; Chen et al., 2012; Tang et al., 2016). 
In the process of environmental risk assessment, the inaccuracy of data, randomness of risk and subjectivity of researchers will lead to a certain degree of bias in the assessment results, which is the uncertainty in environmental risk assessment (Xing et al., 2006; Xu et al., 2009; García-Díaz et al., 2012). The current studies show that the existence of uncertainty makes it impossible to obtain the best estimation of the consequences in a given environmental accident, or the results obtained from analysis are not necessarily the best results. However, the existence of uncertainty does not mean that the risk assessment cannot be carried out; on the contrary, the analysis of uncertainty is an indispensable part of environmental risk system. The uncertainty reflects the integrity of risk information to some extent. There are complex causes of the uncertainty in environmental risk assessment, only by effective screening and classifying, can the corresponding treatment methods be founded out. According to one of the widely recognized classification standard, the uncertainty in environmental risk assessment can be divided into three categories: parameter uncertainty, model uncertainty and the integrity uncertainty (Abrahamsson, 2002). Among them, parameter uncertainty refers to the randomness of the valuing of the parameter; or if the specific value cannot be determined under the given conditions, then the probability distribution interval can be represented. Parameter uncertainty is the main reason for researchers' cognitive bias towards risk results. How to deal with this uncertainty correctly is becoming a hot topic in this research field, and the analysis method based on probabilistic statistics theory is a common research method at present.

The core idea of probabilistic analysis method is to assume that uncertainties are originated from randomness, and by using the probability distribution of one or more variables in the risk system, the uncertainty and variability of the results can be quantified (Maxwell et al., 1999; Abdo et al., 2017). Xu et al. constructed a conceptual model for the evaluation of pollution site exposure process, proposed to characterize the uncertainty of site pollution parameters with probability distribution function, and used Monte Carlo method to assess the contribution of parameter uncertainty to the uncertainty of exposure concentration, thus the probability distribution function of pollutant concentration at exposure points can be established (Xu et al., 2014). As for soil pollution, Labieniec et al. use probability distribution function to describe the uncertainty when estimating the exposure risk of people on the contaminated land. They assessed the uncertainty of carcinogenic risk in contaminated soil due to the uncertainty of geographical characteristics, material destination and migration process (Labieniec et al., 2007).

Through the interpretation of previous research literature, it can be found that it can directly express the transfer process of "Parameter $\rightarrow$ Model $\rightarrow$ Result" by using probabilistic analysis method to deal with the problem of parameter uncertainty (Abrahamsson, 2002; Betrie et al., 2015); but it is noteworthy that the environmental models currently used to realize the quantitative description of pollutant migration is becoming more and more complex, this kind of models often need a lot of input parameters. When using the probability analysis method to carry out the uncertainty analysis, the researchers usually adopt the empirical method and subjective to determine which parameters needed to be involved in probabilistic sampling analysis, and do not consider the contribution of the parameters to the overall uncertainty of the model's output, which would affect the accuracy of the uncertainty analysis results. The flaw of this approach to some extent limit the application and development of uncertainty analysis technology used in environmental risk assessment. So in this paper, we tries to 
introduce sensitivity analysis method to objectively screen out the parameters that have high contribution to the uncertainty of the model calculation result, on this basis, the probability sampling method is used to evaluate the influence of parameter uncertainty on the result of risk assessment so as to provide environmental risk management more scientific and reliable technical support.

\section{Methods}

In order to design a complete environmental risk assessment scheme based on parameter uncertainty analysis, taking the leakage accident of liquid ammonia as a case, the research work in this paper will be carried out in three steps: (1) parameter sensitivity analysis; (2) uncertainty analysis; (3) risk characterization. As shown in Figure 1, firstly, the parameters with statistical characteristic in the accident consequence model are identified, and the parameters' sensitivity analysis are carried out by Morris method so as to screen out a group of parameters that contributed significantly to the uncertainty of the model output; then, these selected parameters are sampled by Latin Hypercube Sampling (LHS) method, the sample data are inputted into the model to simulate, then the probability distribution result of the lethal range values of toxic gas is obtained. Finally, based on the results of uncertainty analysis and the geographic data within the study area, the risk assessment result can be obtained after excuting the spatial analysis in the GIS software.

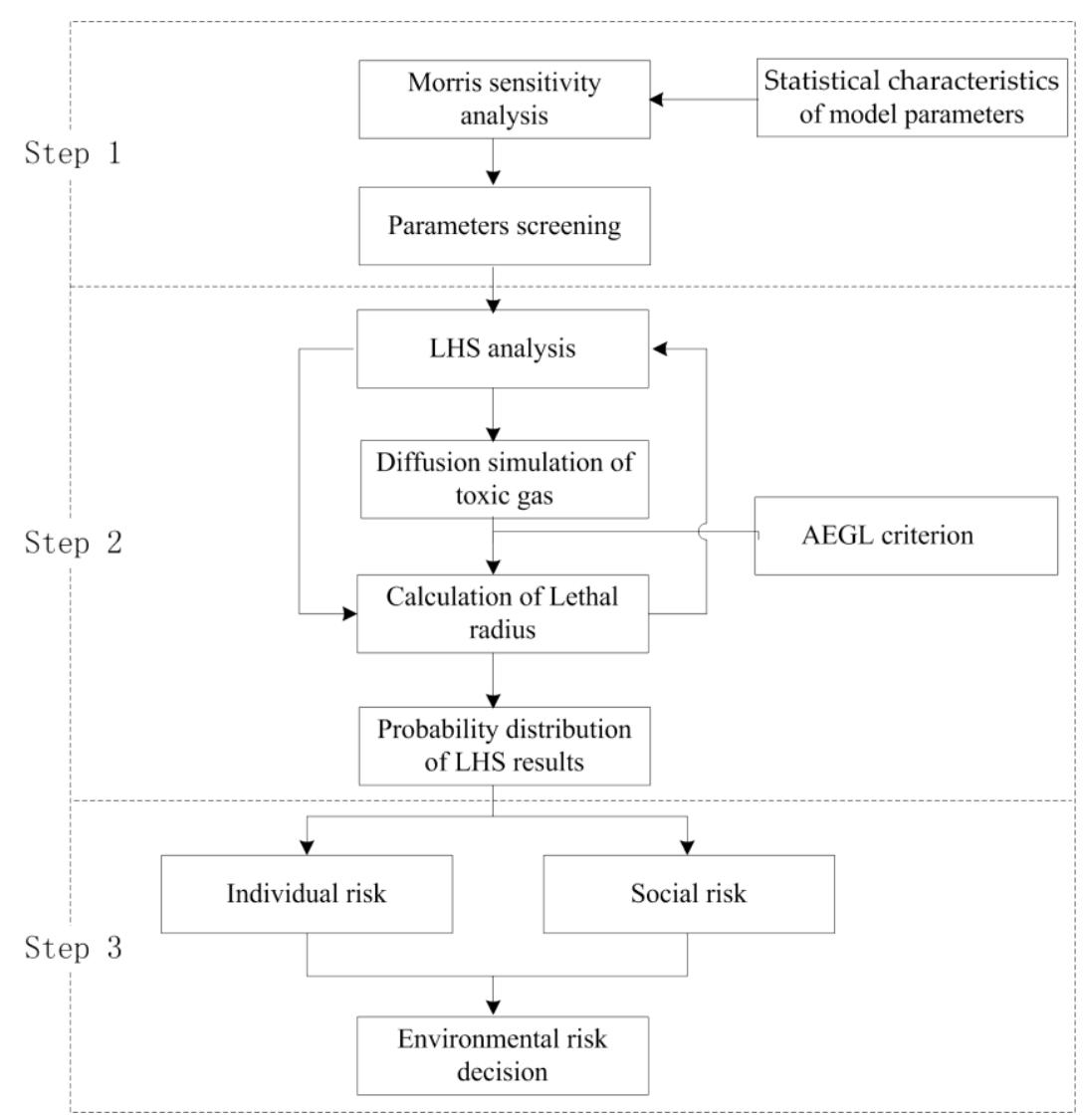

Figure 1. The research scheme of environment risk assessment based on the uncertainty analysis (step 1: parameter sensitivity analysis; step 2: uncertainty analysis; step 3: risk characterization) 


\section{Sensitivity analysis of model parameters}

\section{Accident consequence model and uncertainty parameters identification}

There are many mathematical-physical models that can be used for calculating the harmful effect of hazardous chemical accident, among which, The Process Hazard Analysis Software Tool (PHAST) developed by Det Norske Veritas (DNV) is a very effective software package for quantitative calculation of chemical accident consequence. This software package can simulate the effect degree and spread range of gas leakage, fire and explosion by inputting parameters related at the scene of the accident. The Unified Dispersion Model (UDM) integrated in the PHAST can simulate the diffusion process of gaseous substances. And this model is very complex, which is established on the basis of many classic models including BM model, P-G model (Hanna et al., 2008). PHAST provides the interface with "batch mode", through the Matlab's control program, the repetitive execution of the calculation process can be realized, which provides possibility for subsequent sensitivity analysis and uncertainty analysis (Pandya et al., 2012).

The input parameters of UDM model involve various aspects such as leakage source status, leakage mode, diffusion field parameters and meteorological conditions. By referring to the study of Pandy et al., the parameters with uncertainty in UDM model are identified, the statistical characteristics (probability distribution type and value scope) of these parameters are shown in Table $l$, among which, the parameters' statistical characteristics of leakage source status and leakage mode can be got according to the assumption on the basis of on-site investigation data; the meteorological parameters can be gained based on the statistical analysis to the meteorological data from 2007 2017 of study area, the diffusion field parameter is derived from the expert experience after the users applied PHAST.

Table 1. Statistical characteristics of parameters in PHAST

\begin{tabular}{|c|c|c|}
\hline Parameter name & Meaning/unit & Distribution form \\
\hline $\mathrm{T}_{\mathrm{st}}$ & Storage temperature/K & Triangular(263.15, 283.15, 273.15) \\
\hline $\mathrm{P}_{\mathrm{st}}$ & Storage pressure $/ \mathrm{Pa}$ & Uniform $(0.473 \mathrm{e} 06,0.49 \mathrm{e} 06)$ \\
\hline $\mathrm{L}_{\mathrm{h}}$ & Liquid height/m & Uniform $(12.75,17.25)$ \\
\hline $\mathrm{T}_{\mathrm{a}}$ & Atmospheric temperature/K & $\operatorname{Normal}(251,10.5)$ \\
\hline $\mathrm{P}_{\mathrm{a}}$ & Atmospheric pressure/Pa & Uniform $(0.99 \mathrm{e} 05,1.035 \mathrm{e} 05)$ \\
\hline $\mathrm{H}_{\mathrm{a}}$ & Relative atmospheric humidity/- & Normal $(68,10)$ \\
\hline $\mathrm{u}_{\mathrm{a}}$ & Wind speed $/ \mathrm{m} \cdot \mathrm{s}^{-1}$ & $\operatorname{Normal}(3.4,1.3)$ \\
\hline $\mathrm{D}_{\mathrm{O}}$ & Release orifice diameter/m & Triangular( $(0.16,0.18,0.20)$ \\
\hline $\mathrm{Z}_{\mathrm{R}}$ & Release height above ground $/ \mathrm{m}$ & Uniform $(1,20)$ \\
\hline $\mathrm{Z}_{0}$ & Surface roughness length/m & Uniform $(0.5,1.5)$ \\
\hline$S_{\text {flux }}$ & Solar radiation flux $/ \mathrm{W} \cdot \mathrm{m}^{-2}$ & Triangular $(0,1000,500)$ \\
\hline$\alpha_{1}$ & Jet entrainment parameter & $\operatorname{Normal}(0.17,0.0085)$ \\
\hline$\alpha_{2}$ & Cross-wind entrainment parameter & Normal $(0.35,0.0175)$ \\
\hline $\mathrm{C}_{\mathrm{E}}$ & Cross-wind spreading parameter & Normal $(1.15,0.0575)$ \\
\hline $\mathrm{e}_{\mathrm{pas}}$ & Near-field passive entrainment parameter & $\operatorname{Normal}(1,0.05)$ \\
\hline $\mathrm{C}_{\mathrm{Da}}$ & Drag coefficient of plume in air & Exponential(69.2) \\
\hline$\gamma$ & Dense cloud side entrainment parameter & Exponential(34.6) \\
\hline Ent $_{\text {poo }}$ & Pool vaporisation entrainment parameter & $\operatorname{Normal}(1.5,0.075)$ \\
\hline $\mathrm{r}_{\mathrm{u}}{ }^{\text {pas }}$ & Max cloud/ambient velocity parameter & $\operatorname{Normal}(0.1,0.005)$ \\
\hline
\end{tabular}




\section{Morris sensitivity analysis method}

Sensitivity analysis is used to assess the effect caused by model input parameter on the output result (Xu et al., 2004; King et al., 2013). The qualitative global sensitivity analysis is also called factor screening sensitivity analysis, whose purpose is to gain the rank of each input parameter sensitivity degree in the model through lower computational cost, so that some inessential parameters can be ruled out and the subsequent uncertainty analysis calculation load can be reduced. Morris method was proposed in 1991 by Max D. Morris, which can effectively identify and sort the importance of model parameters. The main idea is to assume that the "Elementary Effect"(EE), which measures the sensitivity of the parameter, is subject to a certain distribution form, and then the global parameter sensitivity can be determined by measuring the mean value and standard deviation value of the distribution (Morris, 1991).

The model contains $\mathrm{k}$ parameters and assumes that the variation range of these parameters is $[0,1]$. Morris sampling method is used to randomly generate a set of initial parameter vectors $\mathrm{X}=\left(\mathrm{x}_{1}, \mathrm{x}_{2}, \ldots, \mathrm{x}_{\mathrm{k}}\right)$, where the value of $\mathrm{x}_{\mathrm{i}}$ is randomly selected from $\{0,1 / \mathrm{p}-1,2 / \mathrm{p}-1, \ldots 1-\Delta\}, \mathrm{p}$ is the number of sampling points, $\Delta$ is the pre-set variable quantity, then the value of $\Delta$ is $\Delta=1 / \mathrm{p}-1$, then the ith parameter's calculation equation of EE are as follows:

$$
E E_{1 i}=\frac{Y\left(x_{1}, \cdots, x_{i}+\Delta, \cdots, x_{k}\right)-Y(X)}{\Delta}
$$

where $\mathrm{Y}(\mathrm{X})$ is the model output corresponding to the initial parameter vector, $\mathrm{Y}\left(\mathrm{x}_{1}, \ldots\right.$, $\left.\mathrm{X}_{\mathrm{i}-1}, \mathrm{X}_{\mathrm{i}}+\Delta, \ldots, \mathrm{x}_{\mathrm{k}}\right)$ is the model output corresponding to the variable quantity $\Delta$ formed by the ith parameter of the initial parameter vector. For the remaining k-1 parameters, repeat the above operation, and calculate the elementary effects of the remaining k-1 parameters respectively and randomly generate $n$ initial vectors. Repeat the above process, then the $n E E$ of $k$ parameters can be represented as $E E_{i j}$, where $j=1,2, \ldots, n$, $\mathrm{i}=1,2, \ldots, \mathrm{k}$. The mean value and standard deviation of the EE of each parameter can be expressed as:

$$
\begin{gathered}
\mu_{i}=\sum_{j=1}^{n} E E_{i j} / n \\
\sigma_{i}=\sqrt{\sum_{j=1}^{n}\left(E E_{i j}-\mu_{i}\right)^{2} / n}
\end{gathered}
$$

\section{The screening of parameters}

The mean value and standard deviation of each parameter can be calculated by Morris method, if the parameter xi corresponds greater mean value $\mu$, then this means it would cause larger influence on the model output value; the larger the value of variance element is, then the larger the interaction is between this parameter and other parameters when affecting the output of the model, or namely, the influence of this parameter to the output of the model is non-linear. Taking these two indexs into 
consideration, $\mathrm{m}$ parameters involved in the subsequent sampling analysis of uncertainty can be screened out.

\section{Uncertainty analysis}

In order to realize the quantitative recognition of the uncertainty of parameters, that is, to describe the risk system by exact numerical value extracted from the conceptual uncertain risk system, the currently most widely used Monte Carlo method can be adopted (Milazzo et al., 2015), which is a kind of calculation method by random sampling statistics to estimate the probability distribution function. Due to Monte Carlo sampling may "collapse" in some sample points and lead to low sampling efficiency, so if the ideal sampling effect wants to be achieved, it requires a large number of sampling test, because of the complexity of the calculation model integrated in PHAST, this process can often take long time with huge calculation resources.

\section{Latin hypercube sampling}

On the basis of Monte Carlo method, Latin Hypercube Sampling (LHS) introduces the idea of multi-dimensional stratified sampling (Hoshino et al., 2000; Wu et al., 2015), that is, $\mathrm{n}$ samples are extracted from m-dimensional vector space. The steps are as follows: each dimension is divided into $n$ intervals that do not overlap each other so that each interval has the same probability; a sample point is randomly selected from each interval in each dimension; then randomly select points from the previous step from each dimension and form them into vectors. As a more efficient sampling method, LHS has greater advantages in both sampling efficiency and running time (due to less number of iterations). Therefore, in this study, LHS is selected as the tool for uncertainty analysis of model parameters. The detailed implementation process in this study is as follows:

1) According to the results of sensitivity analysis (parameters screening), confirm $\mathrm{m}$ uncertain input parameters needed considering and their probability distributions.

2) According to the required sample size $n$, divide the value range of each input parameter $\mathrm{X}_{\mathrm{i}}(\mathrm{i}=1,2, \ldots, \mathrm{m})$ into $\mathrm{n}$ sub-ranges.

3) Randomly select one value in each sub-range from each parameter, in this way, $n$ samples of this input parameter can be obtained. $\mathrm{N}$ samples of $\mathrm{m}$ input parameters are randomly combined to form an $n \times m$ matrix, where $\mathrm{j}(\mathrm{j}=1,2 \ldots, \mathrm{n})$ row represents the input parameters value required for the jth simulation. By substituting the input parameter value matrix into the model, $n$ output results can be obtained.

\section{Conversion to lethal radius}

The PHAST simulation result represents the concentration distribution of diffused toxic gas, while the environmental risk assessment focuses on the impact of toxic substances leakage on human health; Therefore, the concentration result needs to be transformed into the human health effect with the help of the "Toxic dose--Response" reference criteria to get the data needed for environmental risk assessment. In this study, the risk result is shown as the magnitude of risk receptor deaths toll caused by the exposure to the risk area. The minimum lethal dose in the toxicity criteria is selected as the threshold to demarcate the radius of lethal area (hereinafter referred to as "lethal radius"), and the toxic gas diffused range is divided into the lethal area and the nonlethal area. The lethal radius is determined by the short-term exposure guidelines-Acute 
Exposure Guideline Levels (AEGL) developed by National Advisory Committee (NAC) of U.S.

\section{Statistical analysis of LHS result}

After $\mathrm{n}$ times of repeated simulation, and the conversion of the simulation results through AEGL criterion, $\mathrm{n}$ lethal radius values can be obtained. By statistical analysis to the simulation results, the Probability Distribution Function (PDF) and Complementary Cumulative Distribution Function (CCDF) can be obtained, which are important bases of risk characterization.

\section{Risk characterization}

Risk characterization is a process to obtain the final quantitative risk assessment results through spatial analysis in geographic information system, it takes the analysis result of LHS analysis as the base and comprehensively takes into account the factors not involved in LHS analysis, including wind direction probability, accident occurrence probability and population distribution, etc. At present, the regional environmental risk assessment is mainly based on the geographical information system to realize the refined segmentation of the study areas so as to endow the environmental risk with geospatial attributes (Wu et al., 2006; Di Domenico et al., 2014). For the convenience of calculation, the target study area is gridded in the geographic information system platform, and the grid layer of environmental risk source, environmental risk receptor and LHS analysis results included in the risk system is established. According to all types of risk factors, every grid is given an value assignment, so the individual risk and social risk can be calculated respectively with the grid as the calculation unit.

\section{Individual risk}

It refers to the probability of death (or a specific level of injury) to an unprotected risk receptor in a specific location of the risk area for a relatively longer time. Individual risk is usually expressed as the risk contour line, and its calculation method is to calculate the individual risk in each grid center generated by each risk source in the study area. Overlay analysis would be done in the individual risk of each risk source, then the total individual risk value in a single grid can be obtained. The calculation process is shown in Equation 4.

$$
I R_{(x, y)}=\sum_{i=1}^{M} f_{i} \times p_{i}^{W} \times p_{i}^{L} \times v_{i}
$$

where $(x, y)$ represents the coordinate of each grid center, $f_{i}$ is the probability of an accident occurring in the risk source $i, p_{i}^{w}$ is the probability of wind direction that would locate at $(x, y), p_{i}^{L}$ is the LHS analysis result of the ith risk source that would locate at $(\mathrm{x}, \mathrm{y}), \mathrm{v}_{\mathrm{i}}$ is the individual death probability caused by accident happening at ( $\mathrm{x}$, $\mathrm{y}), \mathrm{M}$ is the number of risk sources in this area.

\section{Social risk}

Social risk is on the basis of individual risk to consider the regional population density, and its practical significance is to calculate the probability of a potential 
accident causing more than or equal to a specified number of deaths. The social risk is usually represented by F-N curve, $\mathrm{N}$ stands for death toll in the abscissa, the ordinate is the probability $\mathrm{F}$ of occurring $\mathrm{N}$ or more death toll. The calculation method is shown in Equation 5, and the calculation result is expressed in the form of F-N curve (Hou et al., 2016; Trbojevic et al., 2000).

$$
F=\sum_{y=1}^{S} \sum_{x=1}^{T} I R_{(x, y)} \quad N_{d} \geq N
$$

where $\mathrm{S}$ is the row number of grid in the study area, $\mathrm{T}$ is the column number of grid, and $\mathrm{Nd}$ is the death toll. From the equation, it can be seen that CCDF is need to be used in the calculation process of social risk.

\section{Results and discussion}

\section{Study area}

Wuhu is an important city and shipping hub in the middle reach of Yangtze River. The warehouse storage of Sinopec is built in Tianmenshan Street, Jinghu district along the Yangtze River. Various hazardous substances such as chemical raw materials and intermediate products make this place become a high potential hazard-formative risk source; and its adjacent area is the urban densely-populated district, so this kind of adverse condition will increase the risk degree of accidental chemical disaster. This study takes the leakage accident of liquid ammonia storage tank in this warehouse as an example to carry out the environmental risk assessment. The spatial layout of the study area is shown in Figure 2.

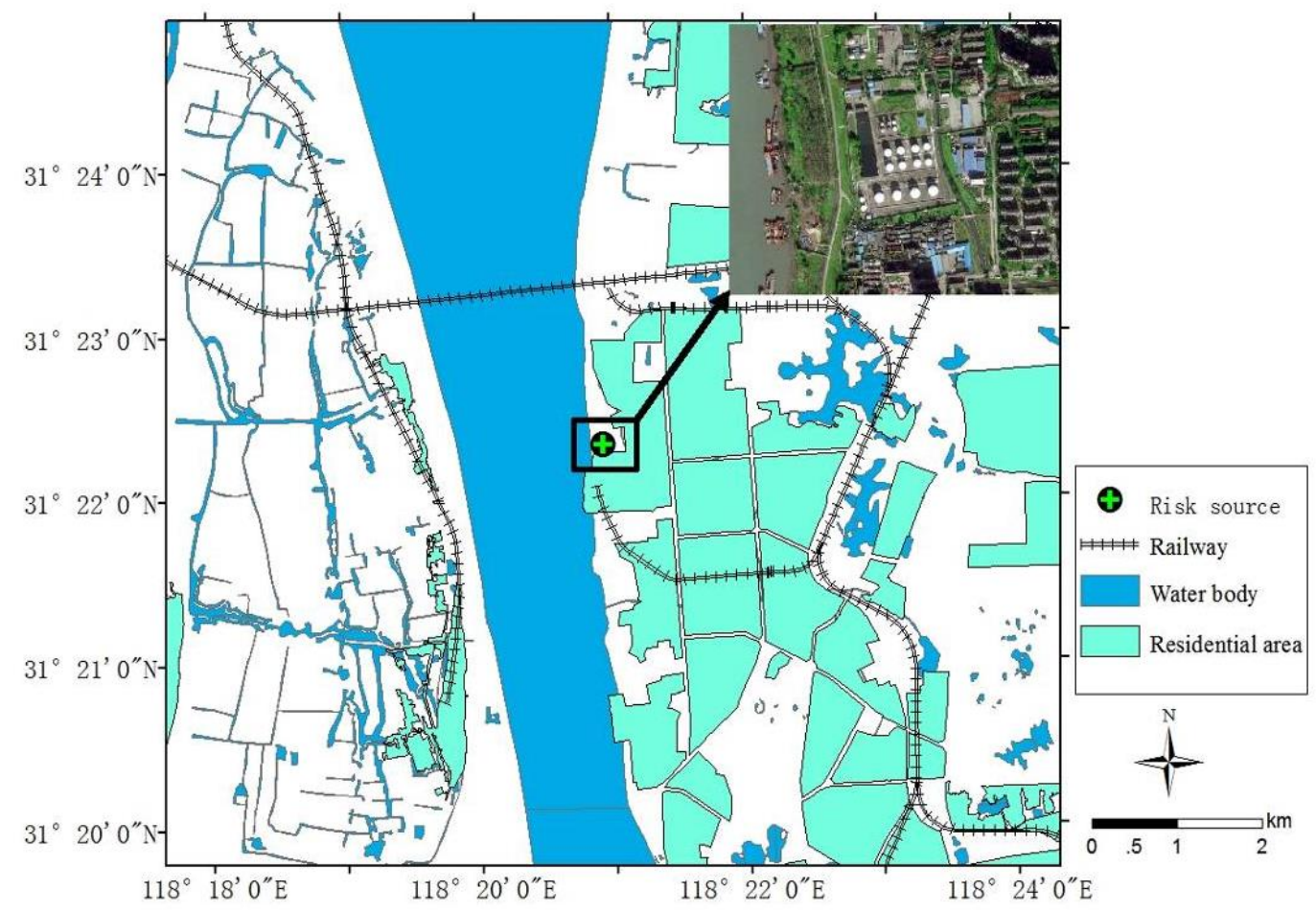

Figure 2. Spatial layout of study area 


\section{Parameters sensitivity analysis}

Ammonia is widely used in industry. It is usually stored in the liquid phase in pressurized vessel. After its emission, a two-phase flow occurs forming an ammonia cloud composed of vapor and very fine droplets that do not fall to the ground. The droplets evaporate quickly, cooling the air. It results a cold mixture of air and ammonia, denser than the ambient air, even though pure gaseous ammonia is lighter than air at ambient temperature (vapor density of 0.73 at $288.15 \mathrm{~K}$ ). For $\mathrm{NH}_{3}$ releases, all the dispersion phases normally occur. Based on the analysis of historical data on leakage accident of liquid ammonia, in research work performed by this paper, the leakage mode is set as "continuous leakage", atmospheric stability class is set as $\mathrm{D}$, with the duration time of $1 \mathrm{~h}$, and the leakage angle is set as horizontal orientation.

According to the principle of Morris method, the sampling of the parameters involved in Table 1 was carried out in 15 orbits and 8 sampling levels, in this way, 120 groups of samples were generated, which were inputted into the PHAST for calculation, and then the ground level ammonia concentration values at $100 \mathrm{~m}, 800$ $\mathrm{m}$ and $3000 \mathrm{~m}$ downwind distance under each group of parameter conditions were respectively calculated, based on which the sensitivity index $\mu$ and $\sigma$ for each parameter are calculated. In order to facilitate the comparison of each parameter's influence degree to ouput result, the scatter diagram shown in Figure $3 a, b$ and $c$ were drawn after taking the normalized values and absolute values of $\mu$ and $\sigma$.

According to the results shown in the scatter diagram, although the sensitivity index of each parameter varies with the downwind distance, on the whole, the parameters which have the highest sensibility are $D_{O}$ (release orifice diameter) and $\mathrm{Z}_{\mathrm{R}}$ (release height above ground). But for the parameters $\alpha_{1}$ and $\alpha_{2}$, the $\mu$ value is higher in the near-field diffusion. This indicates that the effect of jet velocity on ammonia diffusion is higher than wind speed in near-field, while the effect is gradually weakened in far-field diffusion. It can also be seen from the scatter diagram that the $\mu$ value of the parameter is large and the corresponding $\sigma$ value is large generally, this indicates when a parameter has a greater influence on the output of the model, it tends to interact more with other parameters. Here, the $\mu$ values of the parameters corresponding to the three kinds of downwind distances are averaged and ranked. The result is shown in Figure $3 d$. It can be seen that the sensitivity of parameters $D_{0}, Z_{R}, u_{a}, \alpha_{1}, \alpha_{2}, Z_{0}, P_{a}, T_{a}$ have order of magnitudes difference with the remaining parameters in Table 1 . Therefore, they can be considered as parameters that have a greater influence on the output of the model, while other parameters have a very limited influence on the output of the model. Based on this, these 8 parameters are selected to participate in the subsequent uncertainty analysis.

\section{LHS analysis}

LHS is performed on the 8 selected parameters. By referring to the research of Kong et al.(Kong et al., 2011), the sampling times of LHS were set to 1000, and the obtained 1000 groups of parameters value are inputtted into PHAST for simulation, 1,000 simulation results and the PDF of the lethal radius of leakage gas are obtained, CCDF of the lethal radius is also obtained. The detailed results are shown in Figure 4. 


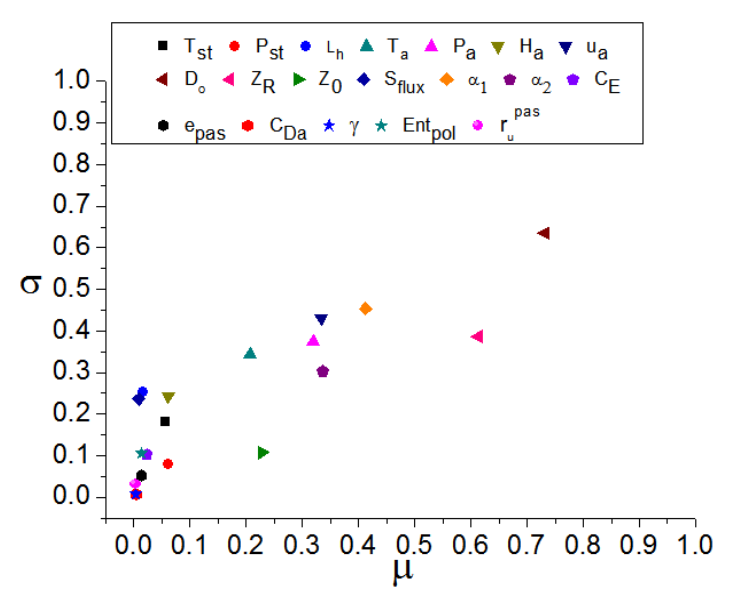

a

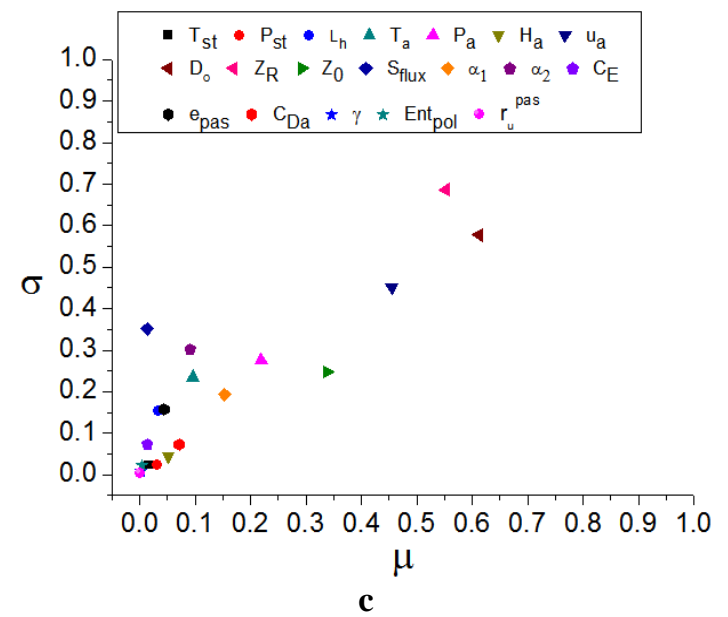

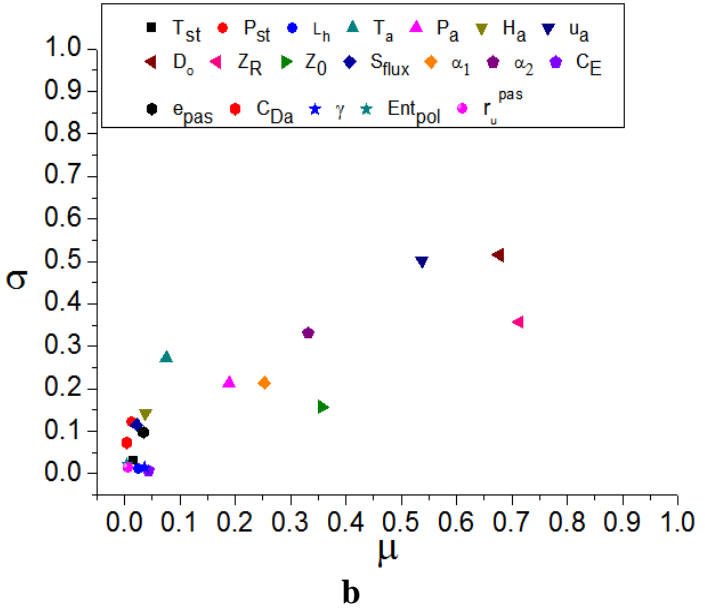

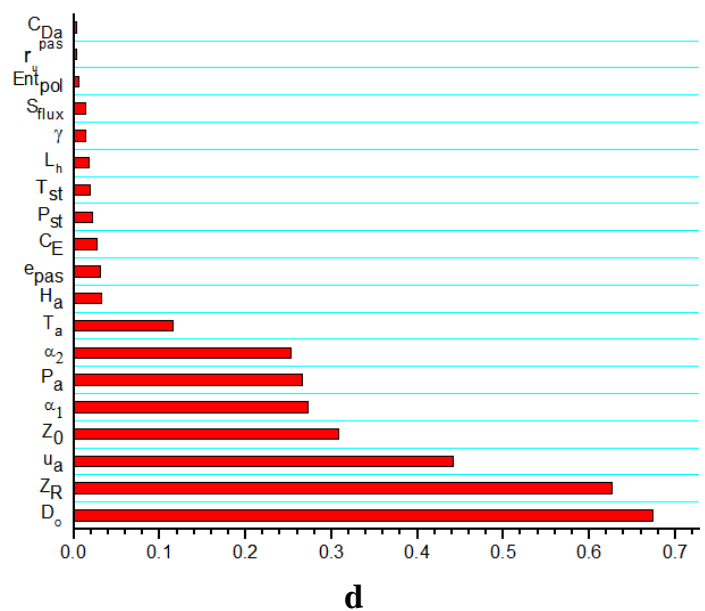

d

Figure 3. Result of parameters sensitivity analysis: (a) Sensitivity index $\mu$ and $\sigma$ at $100 \mathrm{~m}$ downwind distance; (b) Sensitivity index $\mu$ and $\sigma$ at $800 m$ downwind distance; (c) Sensitivity index $\mu$ and $\sigma$ at $3000 m$ downwind distance; (d) Mean value of $\mu$ for all parameters corresponding to the three kinds of downwind distances

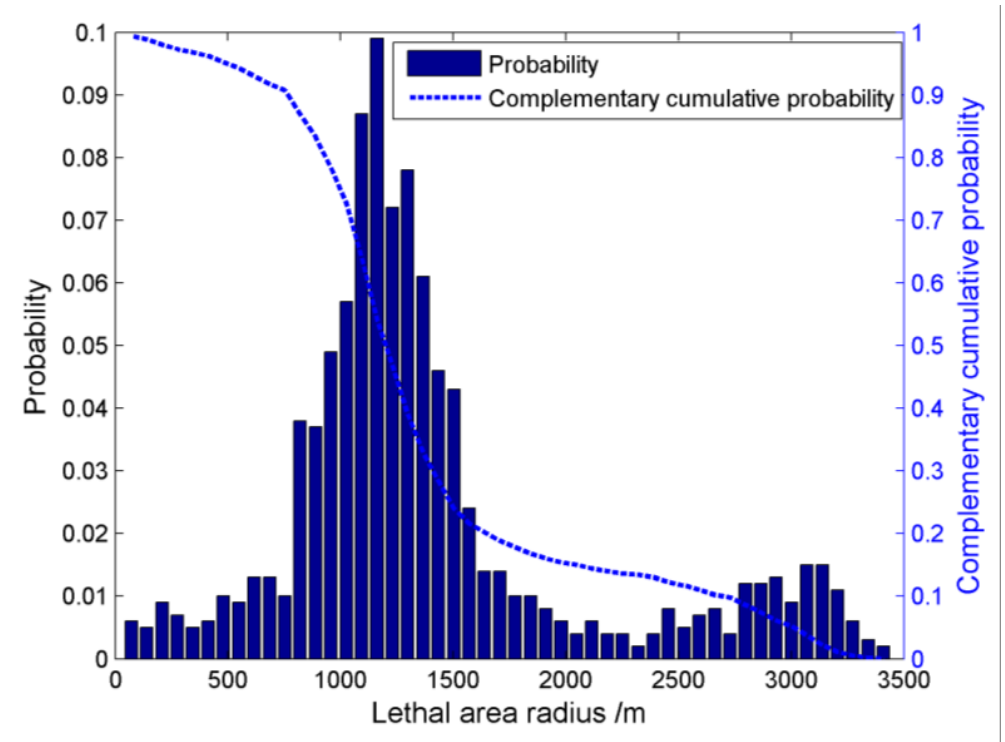

Figure 4. Result of LHS analysis 
As the probability distribution results shown in Figure 4, the lethal radius of liquid ammonia leakage ranges considerably within $100 \mathrm{~m}$ 3500 m under the combined action of 8 independent uncertainty variables in the risk system, among them, the lethal radius around $1300 \mathrm{~m}$ owns greater probability than the ends of this distribution range, which can differ an orders of magnitude. From the analysis results of LHS, two conclusions can be drawn: Firstly, the environmental risk nearly about $1.3 \mathrm{~km}$ distant from the leakage source is relatively high, and the environmental risk beyond $1.3 \mathrm{~km}$ decreases with the increase of distance. Here, the magnitude of environmental risk is only a relative concept based on the LHS results, and the magnitude and acceptability of environmental risk should be evaluated by further introducing other elements in the risk system. Secondly, from the perspective of the lethal concentration distribution, the previously risk assessment research for a single and specific accident scenario is not scientific and reliable enough and would provide incomplete and even wrong information to the researchers.

\section{Risk characterization}

In order to balance the contradiction between the resolution requirement and computational efficiency of risk characterization, the grid scale is set as $50 \mathrm{~m} \times 50 \mathrm{~m}$ in study case considering the actual situation of the study area. The grid partition is carried out in ArcGIS 10.1 to creat the LHS analysis results layer and wind direction probability layer within the study area. The results are shown in Figure 5. The population density of the study area is as shown in the Figure 6. As the grid is used as the calculation unit for environmental risk analysis, all layer files are saved as raster format.

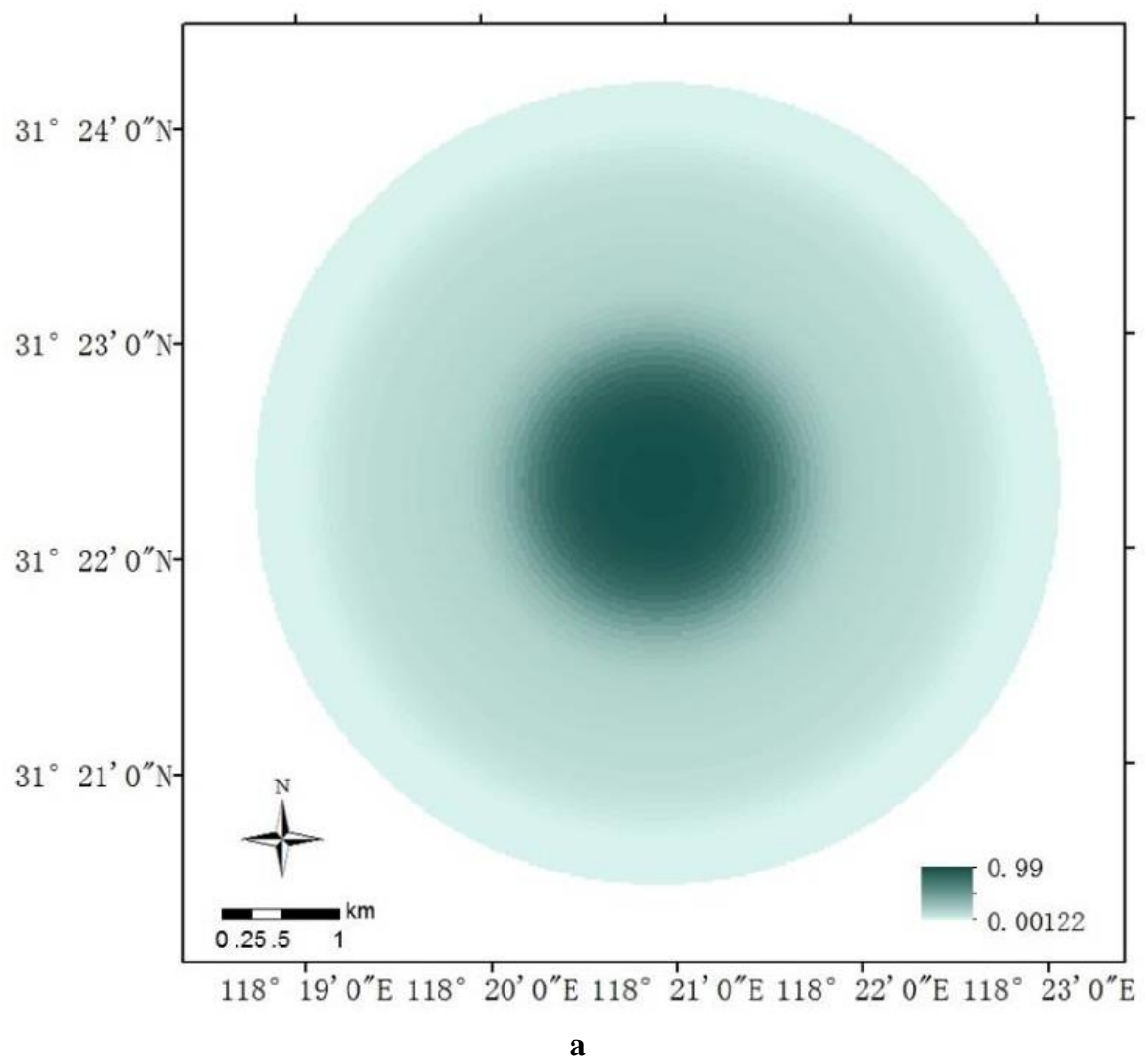




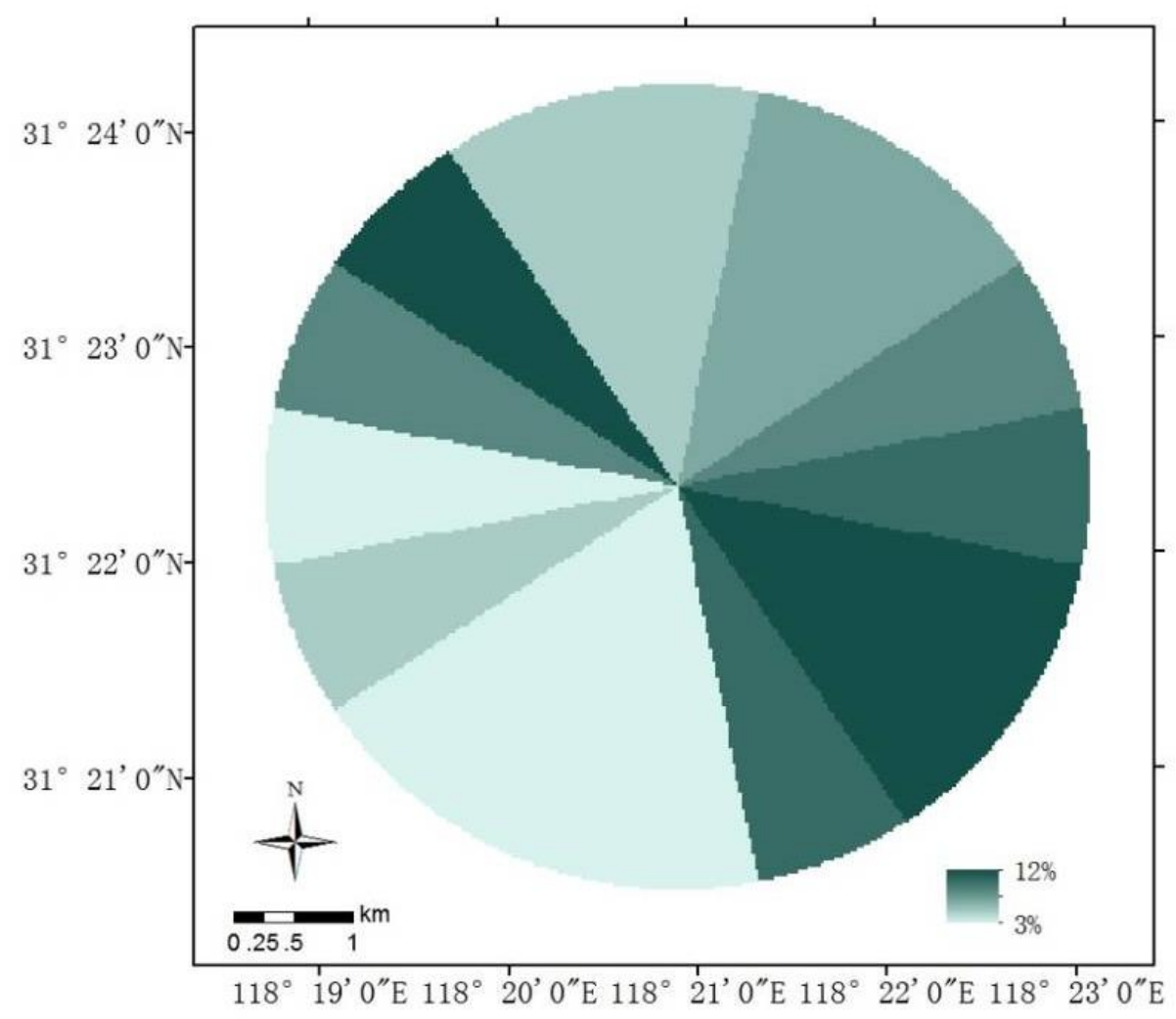

b

Figure 5. Raster layer used in environmental risk assessment. a. Result of LHS analysis. $\boldsymbol{b}$ Wind direction probability

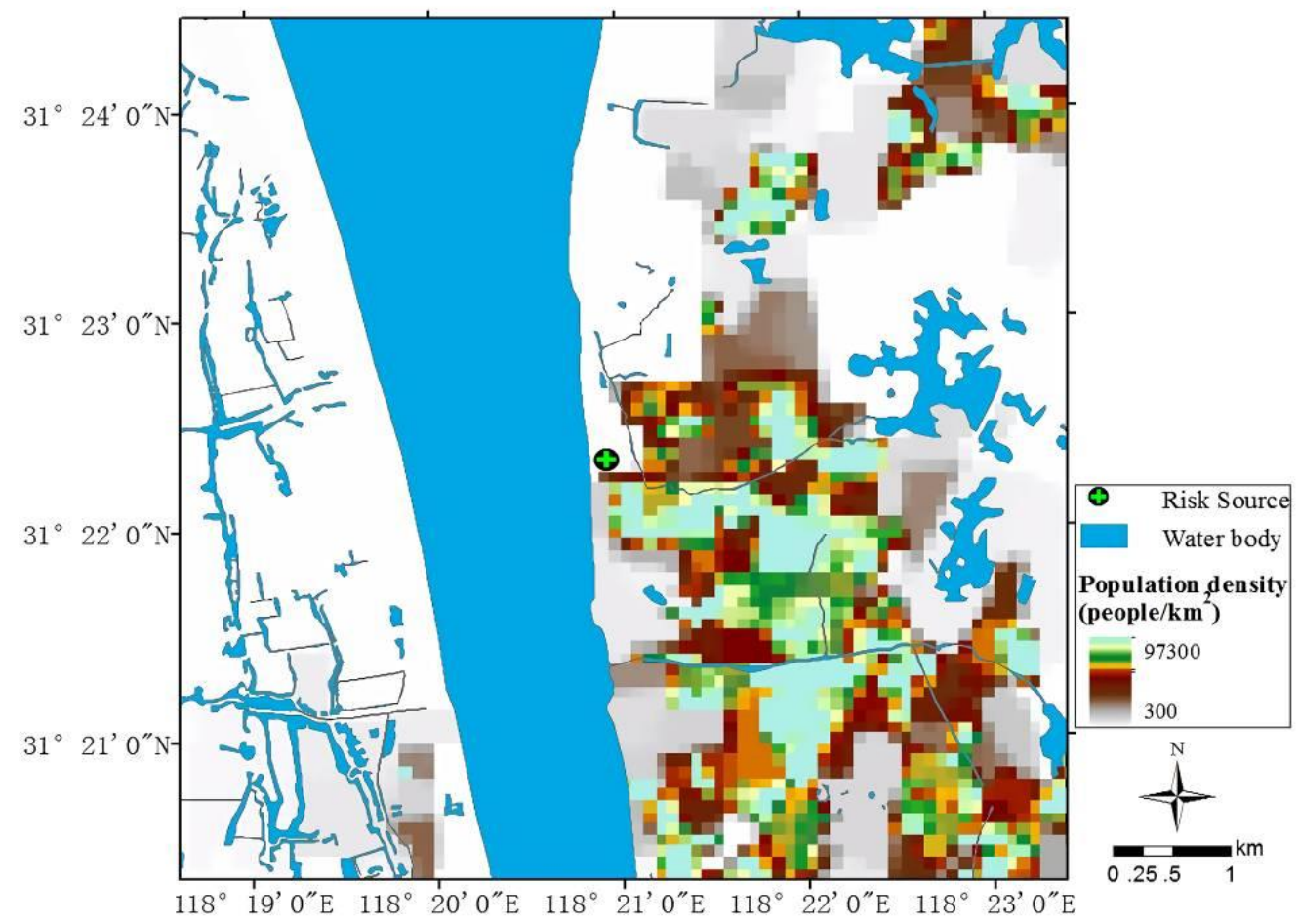

Figure 6. Raster data of population density in study area

APPLIED ECOLOGY AND ENVIRONMENTAL RESEARCH 17(5):11851-11867.

http://www.aloki.hu • ISSN 15891623 (Print) • ISSN 17850037 (Online)

DOI: http://dx.doi.org/10.15666/aeer/1705_1185111867

(c) 2019, ALÖKI Kft., Budapest, Hungary 


\section{Individual risk}

According to the statistical analysis of the liquid-ammonia leakage accident involving more than one fatality in China over the past 20 years, the occurrence probability $\mathrm{f}$ in Equation 4 is about $1.61 \times 10^{-3}$. The CCDF of the lethal radius obtained from the probability sampling analysis is used as the data source of the LHS result layer in ArcGIS. The wind direction probability is obtained through the statistical analysis of historical meteorological data from 2007 2017 in the study area. Since the toxicity criteria used is lethal concentration, so $v$ is set to 1 . On the basis of the discrete results in each grid, the contour lines of individual risk at different levels in the study region can be depicted through grid calculation and extraction analysis in ArcGIS.

After obtaining the assessment results of individual risk, appropriate measure standard should be selected to evaluate the acceptability of risk, which would be used as the basis of environmental risk decision (Meng et al., 2014; Liu et al., 2012). For individual risk, what are now widely recognized standard is As Low As Reasonable Practicable (ALARP) guidelines, set by Health and Safety Executive (HSE) of UK, which also have two kinds of thresholds for individual risk: acceptable risk and negligible risk. This study refers to the HSE standards, also considering the actual construction situation of Chinese hazardous chemical facilities and the socioeconomic performance of assessment object, the threshold of negligible risk level is determined as $10^{-6} /$ year, the threshold of acceptable risk level is $10^{-4} /$ year. Figure 7 shows the individual risk contour lines of these two kinds of thresholds in case study.

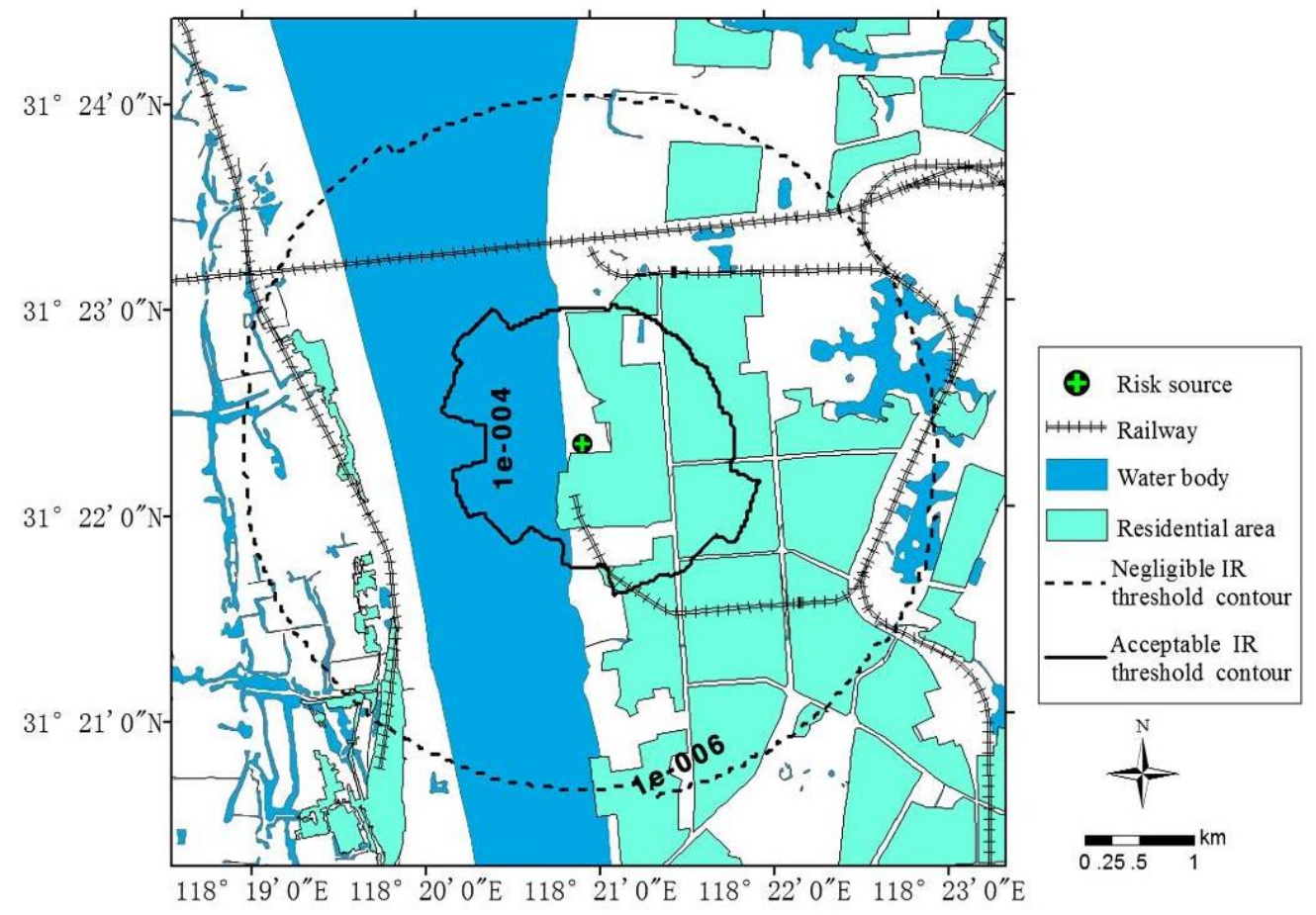

Figure 7. Individual risk assessment result of case study

\section{Social risk}

Social risk is by taking the analysis results of individual risk as the base to calculate the number of risk receptors within the range of individual risk field so as to obtain the 
integrated risk assessment conclusion, and it also follows ALARP criterion. By referring to the measure standard of social risk acceptability in UK and the Netherlands and considering the relatively serious loss of toxic gas leakage accident consequence, the line slope of social risk assessment standard selected in this study is $n=-2$, namely, the risk assessment personnel holds averse attitude; the acceptable line for feature points is determined as $\left(1,10^{-2}\right)$ linear and the negligible line for feature points is determined as $\left(1,10^{-4}\right)$ linear.

The population data of study area is as shown in the Figure 6, hereby, the risk receptor number of each lethal radius can be calculated in ArcGIS, and so the death toll and the corresponding probability can be got. Then according to the calculation results of social risk equation, F-N curve can be depicted and the probability of death toll $(\geq N)$ can be obtained. The details can be seen in the Figure 8 .

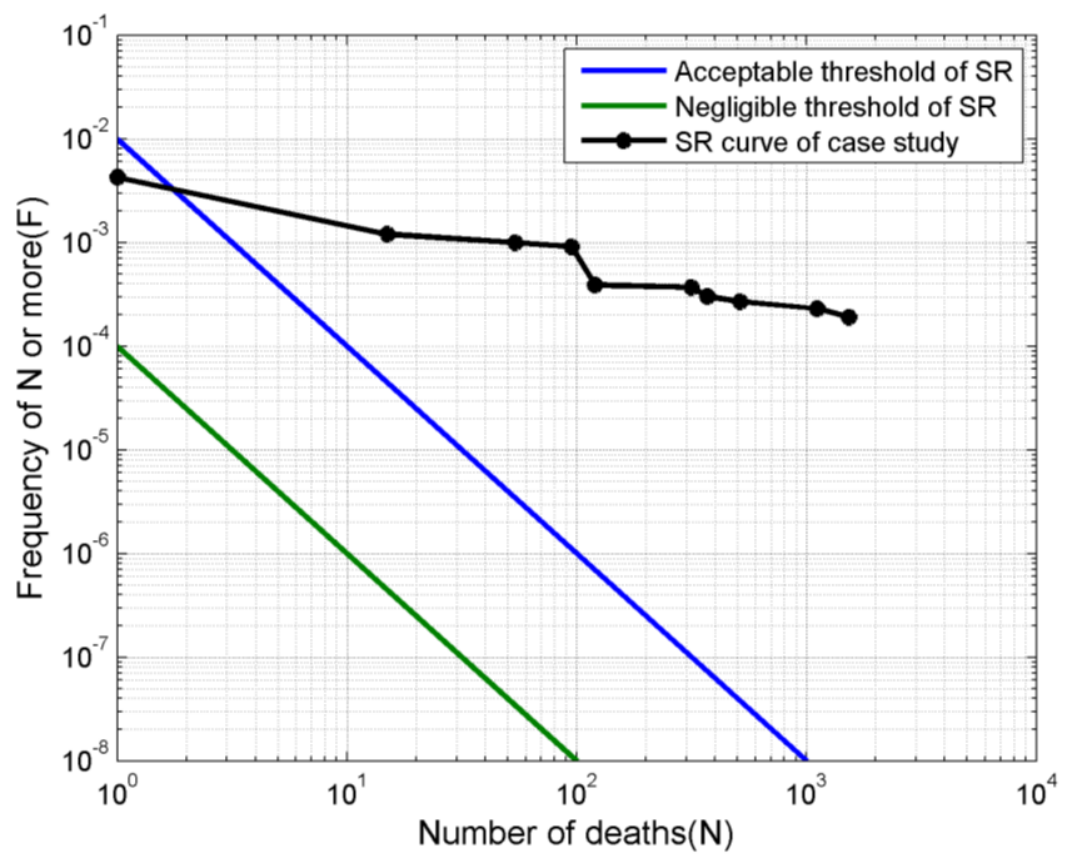

Figure 8. Social risk assessment result of case study

\section{Discussion of environmental risk assessment results}

According to the description of above two sections, the acceptability threshold values of individual risk and social risk are all determined, and based on which we can make some analysis about the environmental risk assessment result.

It can be seen from the individual risk contour line that some residential areas are not in the acceptable range. Through on-site investigation, there are schools and other sensitive public places in the non-ALARP area. Once an accident happens, this part of the population is vulnerable with poor self-rescue ability. According to the principle of ALARP, the relevant departments should take compulsory measures to design and plan this non-ALARP area to reduce the risk level. For people in ALARP area, the government need to further calculate the economic reasonableness before deciding whether to take further measures to reduce the individual risk value in ALARP area.

The F-N curve of case study is mostly above the acceptable level, so the social risk is not acceptable. The highly concentrated residential area near the chemical hazardous 
source is the main reason for this status, namely the unreasonable land use patterns generates higher social risk. This study result also demonstrated a direct relationship between social risk and regional population distribution.

\section{Conclusions}

1) Through the results of parameter sensitivity analysis, it can be found that different input parameters in PHAST would generate different effects on the output results, and some even have magnitude-class differences. The method based on sensitivity sequencing can avoid the subjectivity and blindness of parameter selection in uncertainty analysis. The variability presented of LHS analysis results in this study, in fact, is the expression of the uncertainty of environmental risk system in the reality. Probability sampling method can effectively deal with the uncertainty problems contained in the environmental risk system. The all possible random events can be simulated and finally the probability distribution of the simulation results can be obtained, which become the basis of all potential accident scenarios simulation and more all-sided environmental risk assessment.

2) Based on the uncertainty analysis, individual risk and social risk are used to assess the regional environmental risk level, and the results show that these two kinds of assessment indexes are able to scientifically quantify the environmental risk level as well as visually show the risk level in ArcGIS platform, which contributes to the understanding of the risk decision-makers and provides a reference of risk management for the environmental department. The research results of the liquid ammonia leakage accident show that the individual risk and social risk in the study area are on the high side, which exceed the acceptable threshold. Therefore, corresponding risk management measures should be taken by the chemical enterprise and government to reduce the risk level.

3) It is a common issue concerned by the government and public about the environmental hazard source how to have safe and reasonable layout plan and decide the regional development scale according to the regional environmental risk tolerance in the urban area. In this study, regional environment risk state was evaluated from the aspect of short-term toxic harm for the personnel. Moreover, planning and adjustment suggestions were proposed to the residential area. The follow-up research work can also be in consideration of the long-term impact resulted by environmental accident to soil, water and air. With the combination of uncertainty analysis method proposed in this paper, synthetic judgment was conducted to the environmental risk tolerance for the peripheral zone of environmental hazard source.

Acknowledgements. This study is supported by the "Natural Science Foundation of China" (no. 41671352), "Philosophy Social Sciences Planning Project Of Anhui Province" (no. AHSKY2015D72), and "Science and Technology Planning Project of Huainan, Anhui Province" (no. 2018A361). I would like to thank the anonymous reviewers who have helped to improve the paper.

\section{REFERENCES}

[1] Abdo, H., Flaus, J., Masse, F. (2017): Uncertainty quantification in risk assessment representation, propagation and treatment approaches: application to atmospheric dispersion modeling. - Journal of Loss Prevention in the Process Industries 49: 551-571. 
[2] Abrahamsson, M. (2002): Uncertainty in Quantitative Risk Analysis-Characterisation and Methods of Treatment. - Rep 1024. Lund University Press, Lund, Sweden.

[3] Betrie, G. D., Sadiq, R., Nichol, C., et al. (2015): Environmental risk assessment of acid rock drainage under uncertainty: the probability bounds and PHREEQC approaches. Journal of Hazardous Materials 301: 187-196.

[4] Chen, Y., Song, G. B., Yang, F. L., et al. (2012): Risk assessment and hierarchical risk management of enterprises in chemical industrial parks based on catastrophe theory. International Journal of Environmental Research and Public Health 9: 4386-4402.

[5] Di Domenico, J., Vaz, C. A., de Souza, M. B. (2014): Quantitative risk assessment integrated with process simulator for a new technology of methanol production plant using recycled $\mathrm{CO}_{2}$. - Journal of Hazardous Materials 274: 164-172.

[6] García-Díaz, J. C., Gozalvez-Zafrilla, J. M. (2012): Uncertainty and sensitive analysis of environmental model for risk assessments: an industrial case study. - Reliability Engineering and System Safety 107: 16-22.

[7] Hahn, T., Stauber, J., Dobson, S., et al. (2010): Reducing uncertainty in environmental risk assessment (ERA): clearly defining acute and chronic toxicity tests. - Integrated Environmental Assessment \& Management 5: 175-177.

[8] Hanna, S., Dharmavaram, S, Zhang, J., et al. (2008): Comparison of six widely-used dense gas dispersion models for three recent chlorine railcar accidents. - Process Safety Progress 27: 248-259.

[9] Hansen, S. F. (2016): Environmental risk assessment of chemicals and nanomaterials. Science of the Total Environment 541: 784-794.

[10] Hoshino, N., Takemura, A. (2000): On reduction of finite sample variance by extended Latin hypercube sample. - Bernoulli 6: 1035-1050.

[11] Hou, Z. Q., Zeng, Y. M. (2016): Research on risk assessment technology of the major hazard in harbor engineering. - Procedia Engineering 137: 843-848.

[12] King, D. M., Perera, B. J. C. (2013): Morris method of sensitivity analysis applied to assess the importance of input variables on urban water supply yield - a case study. Journal of Hydrology 477: 17-32.

[13] Kong, D. P., Lu, S. X., Feng, L., et al. (2012): Uncertainty and sensitivity analysis of available safety egress time based on Latin Hypercube Sampling. - Journal of Safety \& Environment 11: 176-179.

[14] Labieniec, P. A., Dzombak, D. A., Siegrist, R. L. (2007): Evaluation of uncertainty in a site-specific risk assessment. - Journal of Environmental Engineering 123: 234-243.

[15] Liu, A. H., Wu, C., Peng, X. (2012): Research on area risk assessment for chemical park based on domino effect model. - Procedia Engineering 45: 47-52.

[16] Lu, Y. S. (1999): Environmental Risk Assessment. - Tongji University Publishing Company, Shanghai.

[17] Maxwell, R. M., Kastenberg, W. E. (1999): Stochastic environmental risk analysis: an integrated methodology for predicting cancer risk from contaminated groundwater. Stochastic Environmental Research Risk Assessment 13: 27-47.

[18] Meng, X. J., Zhang, Y., Yu, X, et al. (2014): Regional environmental risk assessment for the Nanjing Chemical Industry Park: an analysis based on information-diffusion theory. Stochastic Environmental Research and Risk Assessment 28: 2217-2233.

[19] Milazzo, M. F., Vianello, C., Maschio, G. (2015): Uncertainties in QRA: analysis of losses of containment from piping and implications on risk prevention and mitigation. Journal of Loss Prevention in the Process Industries 36: 98-107.

[20] Morris, M. D. (1991): Factorial sampling plans for preliminary computational experiments. - Technometrics 33: 161-174.

[21] Pandya, N., Gabas, N., Marsden, E. (2012): Sensitivity analysis of Phast's atmospheric dispersion model for three toxic materials (nitric oxide, ammonia, chlorine). - Journal of Loss Prevention in the Process Industries 25: 20-32. 
[22] Shao, C. F., Yang, J., Tian, X. G., et al. (2013): Integrated environmental risk assessment and whole-process management system in chemical industry parks. - International Journal of Environmental Research and Public Health 10: 1609-1630.

[23] Tang, C., Yi, Y., Yang, Z., Sun, J. (2016): Risk analysis of emergent water pollution accidents based on a Bayesian network. - Journal of Environmental Management 165: 199-205.

[24] Trbojevic, V. M., Carr, B. J. (2000): Risk based methodology for safety improvements in ports. - Journal of Hazardous Materials 71: 467-480.

[25] Wu, G. J., Chen, W. Z., Tan, X. J., Yang, D. S. (2015): Program development of finite element reliability method and its application based on Latin hypercube sampling. - Rock \& Soil Mechanics 36: 550-554.

[26] Wu, Z. Z., Duo, Y. Q., Wei, L. J., et al. (2006): Quantitative area risk assessment method and its application in land use safety planning for major hazard installations. Engineering Science 8: 46-49.

[27] Xing, K. X., Guo, H. C. (2006): Uncertainty analysis methods in environment model. Environmental Science \& Technology 29: 112-114.

[28] Xu, C. G., Hu, Y., Chang, Y., Jiang, Y., Li, X., Bu, R., et al. (2004): Sensitivity analysis in ecological modeling. - Chinese Journal of Applied Ecology 15: 1056-1062.

[29] $\mathrm{Xu}, \mathrm{H}$. (2009): Advances of the strategies for addressing uncertainty in environmental risk assessment. - Environmental Science \& Management 11: 266-273.

[30] Xu, Y., Liu, J. C., Liu, Y. Q., Neng, C. X., Dong, L. (2014): Quantification of uncertainty in evaluating the health risk of a contaminated site based on Monte Carlo method. - Acta Scientiae Circumstantiae 34: 1579-1584. 